\title{
Postoperative Analgesia with Remifentanil vs Morphine-Metamizole Following Cardiac Surgery
}

\author{
Alfred Ibrahimi ${ }^{*}$, Saimir Kuci ${ }^{1}$, Ervin Bejko ${ }^{1}$, Stavri Llazo ${ }^{1}$, Marsela Goga ${ }^{1}$, Ermal Likaj ${ }^{2}$, \\ Selman Dumani ${ }^{2}$, Ali Refatllari², Jacob Zeitani ${ }^{2}$
}

Received: 12 June 2021 / Accepted: 01 July 2021 / Published online: 20 July 2021

This article is published with open access at https://journal.astes.org.al

(C) The author(s) 2021. \& Copyright (C) 2021, the Albanian Society for Trauma and Emergency Surgery

(c) The Albanian Journal of Trauma and Emergency Surgery is an Open Access Journal. All articles are distributed under the terms of the Creative Commons Attribution Non-Commercial License: http://creativecommons.org/licenses/by-nc/4.0/) which permits unrestricted non-commercial use, distribution, and reproduction in any medium provided the original work is properly cited.

\begin{abstract}
Background: Pain management after cardiac surgery has been based on parenteral long-acting opioids such as morphine. The other alternative is remifentanil. We compared the efficacity of remifentanil vs morphine -metamizole for post cardiac surgery pain relief. Methods; Twenty patients undergoing on-pump coronary artery bypass surgery, receiving standardized propofol-fentanyl and propofol based anesthesia, remifentanil group (Group $\mathrm{R}, \mathrm{n}=10$ ) and fentanyl (Group $\mathrm{F}, \mathrm{n}=10$ ). Postoperative analgesia was provided in $\mathrm{R}$ group initially with remifentanil and later with morphine-metamizole and in F group immediately after operation. Pain was controlled by visual observation, questioning, in rest and during coughing, with a score (0-3).

Results: There is no difference in time of extubation between groups but, pain score was much higher in F (3-9) group in first hour compared with R group (0-4). Morphine requirements was higher in (R) after remifentanil was stopped, in a first hour, but was lower after 24 hours compared with $\mathrm{F}$ group.

Conclusion: Use of remifentanil is associated with lower scale of pain in postoperative period and lower morphine requirement after 24 hours, when analgesia treatment was changed.
\end{abstract}

Keywords: Pain, Remifentanil, morphine-metamizole, cardiac surgery.

Abbreviations: CABG - Coronary Artery Bypass Graft; ICU - Intensive Care Unit; ECG - Electrocardiogram; NSAID - Non-steroidal anti-inflammatory drugs; VPE - Visual Pain Evaluation: HR - Heart Rate; SAS - Systolic Arterial Pressure; MAP - Mean Arterial Pressure: SD - Standard Deviation: CEC - Circulation Extracorporeal; BMI - Body Mass Index; FiO - The fraction of inspired Oxygen; $\mathrm{PaCO}_{2}$ - Partial Pressure of Carbon Dioxide

\section{Introduction}

Postoperative analgesia is a critical risk factor for developing pulmonary and cardiovascular complications in all kinds of thoracic surgery, especially coronary artery bypass graft $(\mathrm{CABG})$ procedures. Patients with elevated pain levels fail to expand their lungs properly, which is called atelectasis.

Original article, no submission or publication in advance or in parallel

\footnotetext{
* Corresponding author:

Alfred Ibrahimi MD, Ph.D.

$\bowtie$ alfredibrahimi@hotmail.com
}

1 Anesthesia service German Hospital Tirana, ALBANIA

2 Cardiac Surgery service German Hospital Tirana, ALBANIA
This may lead to cardiac ischemia and arrhythmia. To maintain patient general health status, well-being, relaxation, and pain management, different sedatives and analgesics are used in the intensive care unit (ICU) as a common strategy. (1-3). Actually, there are too many drugs used in postoperative period for reducing pain: NSAID, ketamine, Dexmedetomidine, magnesium, local anesthetics, gabapentin, epidural anesthesia, (4). Major role in cardiac anesthesia plays high doses of opioids, but can lead to prolonged sedation and delay in tracheal extubation. (5). Introduction of remifentanil as a short acting opioid has changed the course of cardiac anesthesia. Use of remifentanil during anesthesia provides intense analgesia and decreased sympathetic response to surgical stimulation. It has been used to provide sedation in intensive care units. Addition of remifentanil to the perioperative regime may be beneficial with greater 
cardiovascular stability intraoperatively and during sedation immediately postoperatively. (6) The purpose of this study was to determine the effects of remifentanil on postoperative analgesia requirement in patients undergoing open heart cardiac surgery, compared with morphine metamizole analgesia.

\section{Materials and method}

All the patients included in this study had as general criteria: CABG, normal and moderate impaired left ventricular function (ejection fraction $>0.35$ ) and possibility of early extubation in ICU. Patients with severely impaired left ventricular function (ejection fraction $<0.3$ ), significant arrhythmias, evidence of severe congestive heart failure, intra-aortic balloon assist, inotropic support before intervention, and severely impaired major organ function were excluded from this study.

20 patients with all the criteria above were divided in two groups. First group or remifentanil group (R), of 10 patient opioid analgesic of choice during anesthesia and as postoperative analgesic was remifentanil, and second group or fentanyl group (F), of 10 patient opioid analgesic during anesthesia, was fentanyl, and as postoperative analgesics were morphine-metamizole.

Night before operation, all the patient received 2,5 $\mathrm{mg}$ lorazepam. In the next day, in the operating room, all the patient were monitored according to standard guidelines; ECG, pulse oximeter, invasive blood pressure cannulating left radial artery, and central venous catheter with three lumen 7,5 FR Arrow. In the remifentanil group (R), anesthesia was induced using, propofol $3 \mathrm{mg} / \mathrm{kg}$, rocuronium $1,5 \mathrm{mg} / \mathrm{kg}$, and fentanyl $3-4 \mathrm{mcg} / \mathrm{k}$ and analgesia during surgery was maintained with remifentanil $0,1 \mathrm{mg} / \mathrm{kg} \mathrm{min}$. In a fentanyl group (F), the induction dose was $4 \mathrm{mcg} / \mathrm{kg}$. The trachea was intubated $3 \mathrm{~min}$ after induction. The patients were ventilated with oxygen-enriched air (50\% oxygen). The anesthesia was maintained in the first group (R) with remifentanil with maintenance dose $0,2 \mathrm{mcg} / \mathrm{kg} / \mathrm{min}$ and propofol 3-4 mg/kg/min and sevoflurane 1-2 \% in moments of skin incision and median sternotomy. In the second group (F), fentanyl was continued with bolus interval with a max total dose $10-12 \mathrm{mcg} / \mathrm{kg}$. with sevoflurane reinforced. At the end of surgery, patients were kept sedated with propofol $100-200 \mathrm{mg} / \mathrm{h}$, infusion of the study drug was continued and remifentanil. $0,1 \mathrm{mcg} / \mathrm{kg} / \mathrm{min}$ in (R) group and $0,5-$ $1 \mathrm{mg} / \mathrm{h}$ morphine and $1000 \mathrm{mg}$ metamizole was started. Patients were transferred to the cardiac intensive care unit. Tracheal extubation was performed when the patients were cardiovascular stable, were normothermic (central temperature $>36.5 \mathrm{C})$, were not bleeding $(<50 \mathrm{ml} / \mathrm{h})$ and had adequate spontaneous ventilation (tidal volume $>$ $7 \mathrm{ml} \mathrm{kg} 1$, Fio $2<50 \%$ and $\mathrm{PaCO}_{2}<40 \mathrm{~mm} \mathrm{Hg}$ ). In the first group (R), in a first 5 hours after extubation analgesia was maintained with remifentanil, and one hour before it was stopped morphine -metamizole analgesia was started. All the patients' characteristics age, gender, body weight, height and BMI were recorded. Duration of surgery, total duration of postoperative sedation, duration of postoperative intubation (intubation time) and total propofol, remifentanil and fentanyl infused were also recorded. Additional drugs for hemodynamic stability and non-opioid drug to control the pain were taken in consideration.

After extubation for evaluation of pain, we used a Visual Pain Evaluation (VPE). Pain score (0 - 3); if the patient doesn't fill any pain in verbal questioning, is calm and comfortable and does not require any intervention takes 0 score. In agitated patient and distressed, complaining for pain but settles with reassurance and verbal command takes 1 score. If the patient in agitated and require more analgesics, and mild sedation takes 2 score. 3 score takes the patient who cannot be managed by one nurse and requires heavy sedation and bolus of analgesics.

Table 1 Patient characteristics and operative data. Group R, remifentanil; GroupF, fentanyl. Data are presented as mean (SD) or median (range). There were no significant differences between groups

\begin{tabular}{lll}
\hline & GroupR $(n=10)$ & GroupF $(n=10)$ \\
\hline Age(yr) & $62(34-75)$ & $60(48-69)$ \\
Male/female & $7 / 3$ & $6 / 4$ \\
Weight(kg) & $80.9(16.3)$ & $76.5(14.3)$ \\
Height $(\mathrm{cm})$ & $169.5(8)$ & $167.5(6.6)$ \\
Cross clamp time & $32(25-50)$ & $33(26-52)$ \\
CEC time & $40(32-60)$ & $41(33-62)$ \\
Durationofsurgery(min) & $196(52.7)$ & $198(35)$ \\
Totaldurationofstudyinfusion(min) & $499.4(81)$ & $482.7(88)$ \\
Remifentanil dose & $3,3 \mathrm{mg}(2,5-4,6)$ & \\
Fentanyl dose & & $650 \mathrm{mcg}(500-800)$ \\
Propofol dose & $950(800-1200)$ & $1100(950-1500)$ \\
Postoperative sedation time & $75(55-103)$ min & $73(50-99) \mathrm{min}$ \\
Extubation time & $290(35) \mathrm{min}$ & $300(40) \mathrm{min}$ \\
\hline
\end{tabular}




\section{Results}

Both groups had similar characteristics and operation data (Table 1)

In postoperative period, 3 patients of (R) group and 7 patient of $F$ group required nitroglycerine to stabilize the pressure after propofol was stopped. There were no significant differences in HR, MAP and SAP between the two groups before stopping sedation. In Group (F), HR, MAP and SAP increased significantly after stopping sedation. Regarding pain after extubation the $\mathrm{F}$ group tender to be much higher than in $\mathrm{R}$ group Table 2 . The patient in R group in a first 8 hour doesn't fill any pain (remifentanil-controlled analgesia), but after cessation e remifentanil this pain increased (score 1), but in fentanyl group the pain decreased from score 2 to one, and in second postoperative day all the patient is both groups the pain score 0 . Regarding use of other analgesics (acetaminophen $600 \mathrm{mg}$ iv), and bolus of morphine was applied in 6 patients in first 2 hour after extubation, and no one to (R) group.

\section{Discussion}

In this study we are focused to resolve two issues in our institution. First, fast truck anesthesia, and second postoperative protocol for pain management. There is a connection between two issues, because to achieve the first one we have to reduce the dose of fentanyl in group (F) from $20 \mathrm{mcg} / \mathrm{kg}$ to $10-12 \mathrm{mcg} / \mathrm{kg}$. Regarding the time of extubation there is no difference between two groups. Khanykin and colleges [7] in their study have concluded that remifentanil fast-track anesthesia for cardiac patients has no negative impact on myocardial function. Both remifentanil and low-dose fentanyl are equally effective and safe for fast-track cardiac anesthesia. This is in correlation with other study $[8,9,10]$. The study did not highlight any statistical superiority of remifentanil anesthesia over lowdose fentanyl anesthesia. Reducing the dose of fentanyl for achieving fast truck anesthesia was associated with increase a intense pain, especially in a first hour in a (F) group (pain score 3-9) and was associated with elevated HR and SAP. Bolus of 2-3 mg morphine and 50-100 mg metamizole was applied to reduce the pain and nitroglycerine iv for controlling blood pressure ( 7 patients).

In $\mathrm{R}$ group, intense of pain was lower during an infusion of remifentanil in analgesic dose, (pain score 0-3), but intensity is increased after remifentanil was stopped and morphine- metamizole sedation was started. But intense of pain was much lower than in first hour in a (F) group after extubation, requirement of morphine in (R) group initially was higher but in 24-hour interval was lower than in group (F). In a study [10] with the same number of patient and with the same characteristics they concluded that remifentanil is associated with increased analgesic requirements during the first $30 \mathrm{~min}$ to $1 \mathrm{~h}$ after cessation following prolonged use

Table 2 Results showing changes in heart rate (HR), systolic arterial pressure(SAP) and mean arterial pressure (MAP) before (pre-) and after (post-) stoppingpropofol. Data are presented as mean (SD).

\begin{tabular}{lll}
\hline & Group $R(n=10)$ & Group $F(n=10)$ \\
\hline $\begin{array}{l}\text { No. of patients requiring drugs } \\
\text { to stabilize haemodynamics }\end{array}$ & 3 & 7 \\
& & \\
HR pre-sedation & $74(15)$ & $73(7)$ \\
HR post-sedation (beats/min) & $80(15)$ & $95(8)$ \\
SAP pre-sedation (mmHg) & $105(18)$ & $125(10)$ \\
SAP post-sedation (mmHg) & $115(12)$ & $135(8)$ \\
MAP pre-sedation (mmHg) & $65(19)$ & $83(9)$ \\
MAP post-sedation $(\mathrm{mmHg})$ & $75(6)$ & $89.5(6)$ \\
\hline
\end{tabular}

Table 3. Pain scores, in a first hours, and after 6 and 24 hours, other analgesic consumption.

\begin{tabular}{lll}
\hline & GroupR $(n=10)$ & GroupF $(n=10)$ \\
\hline Pain score (VPE) 1 hour & $2(0-4)$ & $6(3-9)$ \\
Pain score 6 hour & $2(0-4$ & $1(0-3)$ \\
Pain score after 24 hours & $1(0-2)$ & $1(0-2)$ \\
Morphine bolus (2 hour) & & 6 \\
Pain after coughing & 2 & 7 \\
Acetaminophen $600 \mathrm{mg}$ & 2 & 6 \\
\hline
\end{tabular}


( $>8 \mathrm{~h}$ ). the intense of pain, morphine requirements, propofol consumption was much lower than in this study above. The difference between our study and the study (10) is the use of fentanyl as induction of anesthesia and shorter remifentanil infusion (6 hour) after extubation.

\section{Conclusion:}

Use of remifentanil is associated with lower scale of pain in postoperative period and lower morphine requirement after 24 hours.

COI Statement: This paper has not been submitted in parallel. It has not been presented fully or partially at a meeting or podium or congress. It has not been published nor submitted for consideration beforehand.

This research received no specific grant from any funding agency in the public, commercial, or non-profit sectors. There are no relevant or minor financial relationships from authors, their relatives or next of kin with external companies.

Disclosure: The authors declared no conflict of interest. No funding was received for this study.

Acknowledgement: We would like to thank medical staff of Cardiac Surgery \& Anesthesia Service German Hospital Tirana, ALBANIA

\section{References}

1. Bair N, Bobek MB, Hoffman-Hogg L, Mion LC, Slomka J, Arroliga AC. Introduction of sedative, analgesic, and neuromuscular blocking agent guidelines in a medical intensive care unit: physician and nurse adherence. Crit Care Med. 2000;28(3):707-713.

2. Berger JT, Rosner F. The ethics of practice guidelines. Arch Intern Med. 1996;156(18):2051-2056. doi:10.1001/ archinte.1996.00440170053006
3. Dasta JF, Fuhrman TM, McCandles C. Patterns of prescribing and administering drugs for agitation and pain in patients in a surgical intensive care unit. Crit Care Med. 1994;22(6):974980. doi:10.1097/ 00003246-199406000-00016

4. Analgesia and sedation post-coronary artery bypass graft surgery: a review of the literature Mansour Jannati and Armin Attar Ther Clin Risk Manag. 2019; 15: 773-781. Published online 2019 Jun 20. doi: 10.2147/TCRM.S195267

5. Myles PS, Hunt JO, Fletcher H, Watts J, Bain D, Silvers A. Remifentanil, fentanyl, and cardiac surgery: a double-blinded, randomized, controlled trial of costs and outcomes. Anesth Analg 2002; 95: 805-1

6. Lehmann A, Zeitler C, Thaler E, Isgro F, Boldt J. Comparison of two different anesthesia regimens in patients undergoing aortocoronary bypass grafting surgery: sufentanil-midazolam versus remifentanil-propofol. J Cardiothoracic Vasc Anesth 2000; 14: 416-20

7. Boris Khanykin, Rizwan Siddiqi, Per F Jensen, Dennis R Bigler, Gennady V Atroshchenko Comparison of remifentanil and low-dose fentanyl for fast-track cardiac anesthesia: a prospective randomized study Heart Surg Forum 2013 Dec;16(6):E3248 doi:10.1532/HSF98.2013229.

8. Howie MB, Cheng D, Newman MF, et al. A randomized doubleblinded multicenter comparison of remifentanil versus fentanyl when combined with isoflurane/propofol for early extubation in coronary artery bypass graft surgery. Anesth Analg 2001; 92:1084-93

9. Engoren M, Glenn L, Fenn-Buderer N. A comparison of fentanyl, sufentanil, and remifentanil for fast-track cardiac anesthesia. Anesth Analg 2001; 93: 859-64

10. Coriat $\mathrm{P}$, Beaussier M. Fast-tracking after coronary artery bypass graft surgery. Anesth Analg 2001; 92: 1081-3

11. Remifentanil infusion in association with fentanyl-propofol anaesthesia in patients undergoing cardiac surgery: effects on morphine requirement and postoperative analgesia K. Rauf, A. Vohra*, P. Fernandez-Jimenez, N. O'Keeffe and M. Forrest British Journal of Anaesthesia 95 (5): 611-15 (2005) doi:10.1093/bja/aei237 Advance Access publication September 9,2005 